\title{
Zurückweisung von Verantwortung als Hindernis nachhaltiger Bereitschaften
}

Monika Baier

\section{Zusammenfassung}

Vor dem Hintergrund des anthropogenen Klimawandels und dessen Folgen, steht die Dringlichkeit nachhaltigen Handelns außer Frage. Trotz des öffentlichen Bewusstseins dieser Notwendigkeit, handeln Menschen jedoch nicht automatisch nach haltig. Im vorliegenden Beitrag wird der Einfluss von Verantwortungszuschreibungen und Rechtfertigungsargumenten als Form der Verantwortungsabwehr auf nachhaltige Engagement- und Handlungsbereitschaften thematisiert. Mittels Fragebogenstudie wurden die Daten von 312 Personen erhoben. Die Befunde zeigen, dass Rechtfertigungsargumente hervorgebracht werden, um Maßnahmen zur Einsparung von Energie abzuwenden. Es besteht zudem ein hochsignifikanter, negativer Zusammenhang zwischen Rechtfertigungsargumenten und internaler Verantwortungsattribution. Ferner kann gezeigt werden, dass Rechtfertigungsargumente eine hemmende Wirkung auf nachhaltige Engagement- und Handlungsbereitschaften haben.

\section{$1 \quad$ Zurückweisung von Verantwortung}

Menschliches Handeln bleibt nicht selten gerade dann aus, wenn es besonders dringend erforderlich wäre. Wenn beispielsweise jemand auf einem öffentlichen Platz vor den Augen zahlreicher Passanten attackiert wird, kann es durchaus vorkommen, dass keiner dieser Passanten eingreift. Ursächlich für ausbleibendes Hilfeverhalten können eine Reihe situativer oder dispositionaler Variablen sein, so die Befunde empirischer Studien (Darley \& Batson, 1973). Auf das Beispiel bezogen, mangelt es möglicherweise den unbeteiligten Passanten an entsprechenden

(C) Der/die Autor(en) 2017

K.-D. Altmeppen et al. (Hrsg.), Nachhaltigkeit in Umwelt,

Wirtschaft und Gesellschaft, DOI 10.1007/978-3-658-14439-5_8 
Fähigkeiten einzugreifen oder sie fürchten sich vor negativen Konsequenzen für das eigene Wohlergehen. Ebenfalls denkbar wäre, dass sie aufgrund der ausbleibenden Reaktionen der übrigen Personen zu einer fehlerhaften Einschätzung der Situation gelangen (Darley \& Latané, 1968). Denn das Verhalten oder auch bereits die bloße Anwesenheit anderer spielt eine ganz wesentliche Rolle: Sind andere Personen anwesend, die ebenfalls eingreifen könnten, kann dies zu einer Verantwortungsdiffusion führen, die das eigene Eingreifen unwahrscheinlicher macht, wobei die Anzahl der Zuschauer sowie das Verhältnis, in dem die betreffenden Personen zueinander stehen, ebenfalls einen Einfluss hat (Latané \& Rodin, 1969). In der Psychologie spricht man vom Bystander-Effekt (Darley \& Latané, 1968). Es gibt zahlreiche Untersuchungen zur Verantwortungsdiffusion, in denen belegt wird, dass Personen gewisse Situationen umdeuten, um die Verantwortung dafür abzugeben oder zu verringern, und gleichzeitig das Gefühl einer Verpflichtung ablegen, um nicht handeln zu müssen (Bennett, Banyard \& Garnhard, 2014; van Erp, Rispens, Gevers \& Demerouti, 2015; Pittinsky \& Diamante, 2015). Entwicklungspsychologische Forschungsergebnisse zeigen sogar, dass der Bystander-Effekt bereits bei kleinen Kindern auftritt (Plötner, Over \& Tomasello, 2015).

Ein Modell, in dem diese Zurückweisung von Verantwortung eine zentrale Rolle einnimmt, ist das Normaktivationsmodell von Schwartz (1977). Auch hier gingen Studien zum Bystander-Effekt voraus (Schwartz \& Clausen, 1970). Die Verantwortungszurückweisung in dem besagten Modell stellt die individuelle Tendenz dar, die persönliche Verantwortung für die Folgen des eigenen Handelns abzulehnen (Schwartz \& Howard, 1981). Konkret heißt das, Zurückweisung von Verantwortung und Rechtfertigung bewirken das Ausbleiben eines Verhaltens, selbst wenn die übrigen Komponenten entsprechend ausgeprägt bzw. , aktiviert“ sind. Diese Komponenten setzen sich zusammen aus vorhandener Problemwahrnehmung infolge einer entsprechenden Verantwortungszuschreibung, Bewusstheit der Handlungskonsequenzen, Wirksamkeitserwartungen, Fähigkeit zum Eingreifen, Wahrnehmung kausaler Zusammenhänge sowie Appellen zum Eingreifen, was wiederum abhängig von der persönlichen Norm zu einem aktuellen Gefühl moralischer Verantwortung führt. Aus diesem Gefühl moralischer Verantwortung heraus würde nun gemäß dem Modell das entsprechende Verhalten resultieren, vorausgesetzt, es findet keine Zurückweisung von Verantwortung bzw. Rechtfertigung statt (Schwartz, 1977). Kommt es jedoch zur Zurückweisung, so bewirkt dies, dass das Individuum das Verhalten abwendet, selbst wenn die übrigen Bedingungen dafür gegeben wären. 


\section{$2 \quad$ Handlungsfeld und Ableitung eigener Untersuchungsfragen}

Auch wenn das einleitende Beispiel dem Bereich prosozialer Verhaltensbereitschaften entstammt, so ist das Normaktivationsmodell nicht auf ein spezifisches Verhalten beschränkt, sondern es kann auf verschiedene Verhaltensbereiche, beispielsweise auf Umweltverhalten, übertragen werden. So untersuchten beispielsweise van Liere \& Dunlap (1978) mittels des Modells erfolgreich die Interaktionen der Variablen bezogen auf die umweltschädigende Verbrennung von Gartenabfällen. Gerade die Verantwortungszuschreibung in Kombination mit Gefahrenbewusstsein hatte eine bedeutsame Auswirkung auf das Verhalten. War beides hoch ausgeprägt, so ging es mit dem geringsten Verbrennungsverhalten einher, bei geringer Verantwortung hingegen war das angegebene umweltschädigende Verhalten weitaus stärker ausgeprägt, als bei hoher Verantwortung, selbst wenn das Gefahrenbewusstsein in beiden Fällen hoch ausgeprägt war. Aber auch in anderen Kontexten zeigte sich das Modell bezogen auf umweltrelevante Verhaltensentscheidungen als bedeutsam, beispielsweise bei der Wahl des Verkehrsmittels (Hunecke, Blöbaum, Matthies \& Höger, 2001; Klöckner \& Blöbaum, 2010).

Dass das Empfinden von Verantwortung eine Schlüsselrolle bei umweltrelevanten Handlungen spielt, geht auch aus dem spezifischeren Modell Verantwortlichen Umwelthandelns (Kals, 1996) hervor, welches unter anderem einen starken konzeptuellen Bezug zum Normaktivationsmodell aufweist. Sich für einen Sachverhalt verantwortlich zu fühlen, führt in der Regel zu einer erhöhten Bereitschaft, sich für den Umweltschutz einzusetzen oder umweltschädigendem Verhalten entgegenzusteuern (Kals, Montada, Becker \& Ittner, 1998).

Dieser Verantwortungsaspekt, der sowohl im Normaktivationsmodell von Schwartz, als auch im Modell Verantwortlichen Umwelthandelns von Kals eine zentrale Rolle einnimmt, soll aufgrund seiner hohen Relevanz Gegenstand des vorliegenden Beitrags sein. Hierbei soll vor allem Verantwortungszurückweisung in Form von Rechtfertigungsargumenten als Teilaspekt dieser Modelle näher betrachtet werden.

Von besonderem Interesse ist dabei für die folgende Untersuchung das Handlungsfeld nachhaltiger Verhaltensweisen. Das „unscharfe Prädikat“ Nachhaltigkeit (Linneweber, 1998) wird in diesem Zusammenhang bezugnehmend auf den ökologischen Bereich verwendet, konkret wird umweltrelevantes Verhalten thematisiert. Dieser Aspekt steht meist im Zentrum psychologischer Forschung zur Nachhaltigkeit, nicht zuletzt deshalb, weil hier sehr gute Einflussmöglichkeiten bestehen (Gifford, 2007; Kazdin, 2009). Als spezifisches Handlungsfeld dient die Nutzung und der Konsum von Energie aus fossilen Brennstoffen, denn dies eignet 
sich in besonderer Weise, um die Grundproblematik bei Umweltangelegenheiten zu verdeutlichen und zwar in doppelter Weise. Auf der einen Seite steht die Endlichkeit dieser Ressourcen. Die Menge fossiler Brennstoffe, die aktuell jährlich verbraucht wird, entspricht einem Umfang, für dessen Entstehung in etwa zwei Millionen Jahre notwendig waren. Laut Prognosen werden diese Vorräte je nach Energieträger in 50 bis 150 Jahren verbraucht sein (Girardet \& Mendonça, 2010). Auf der anderen Seite ist die Verbrennung fossiler Energieträger als nicht nachhaltig anzusehen, da sie mit einem sehr beachtlichen Ausstoß klimaschädigender Treibhausgase, vor allem $\mathrm{CO}_{2}$, einhergeht (World Wide Fund For Nature, o. J.). Dies wiederum trägt zur globalen Erwärmung bei, welche nicht zuletzt die Bedürfnisse zukünftiger Generationen, aber auch Menschen in anderen Ländern zu gefährden droht. Hier findet folglich eine zeitliche und räumliche Verschiebung negativer Konsequenzen statt, denn der Leidtragende ist nicht gleichzeitig der Verursacher von Umweltschäden (Pawlik, 1991). Gerade deshalb haben sich bereits zahlreiche Umweltpsychologen dieses Themas angenommen (Linneweber, 1995; Wortmann, 2004), aber es besitzt aufgrund der Dringlichkeit immer noch hohe Brisanz, auch für weitere Forschung (Stern, 2011). Die Folgen der globalen Erwärmung stellen eine ernste Bedrohung dar, zum einen ganz unmittelbar durch die Zunahme von Naturkatastrophen, aber auch durch die Veränderung des Lebensraums, womit nicht zuletzt soziale und gesundheitliche Konsequenzen einhergehen (Fritsche, Cohrs, Kessler \& Bauer 2012; Haines, Ebi, Smith \& Woodward, 2014; Raffa, Eltoukhy \& Raffa, 2012).

Vor allem hinter der räumlich-zeitlichen Verschiebung verbirgt sich eine grundlegende Gerechtigkeitsproblematik. Daher mag es nicht überraschen, dass vielfach nachgewiesen werden konnte, dass die Wahrnehmung von Ungerechtigkeit eine ganz wesentliche Auswirkung auf umweltschützendes Verhalten hat (Clayton, 2000; Müller, 2012; Nancarrow \& Syme, 2001). Allerdings ist es bei sogenannten sozioökologischen Dilemmata oftmals charakteristisch, dass umweltschädigendes Verhalten letztlich erst in der Summe zu den unerwünschten Konsequenzen führt, was im Umkehrschluss bedeutet, dass einzelne Verzichtleistungen oder Handlungen zum Schutze der Umwelt keinen unmittelbar positiven Effekt im Sinne eines Erfolgserlebnisses mit sich bringen (Hardin, 1968; Spada \& Opwis, 1985).

Hier kommen wieder die Verantwortungszurückweisung und die Rechtfertigungsargumente ins Spiel: Auch wenn unter Umständen zwar der Handlungsbedarf erkannt wird, was ein Gefahrenbewusstsein bedeutet, so kann es sein, dass das Individuum für sich selbst Argumente zurechtlegt, beispielsweise basierend auf mangelnden Selbstwirksamkeitserwartungen, was wiederum dazu führt, dass kein entsprechendes Verhalten gezeigt wird. Im Folgenden wird angenommen, dass sich dieser Aspekt aus dem Modell von Schwartz auch im Umweltbereich hinsichtlich 
energierelevanter Entscheidungen abbilden lässt, dass es Zusammenhänge mit verschiedenen Verantwortungsattributionen gibt und dass sich stark ausgeprägte Rechtfertigungsargumente negativ auf energieschützende Handlungsbereitschaften auswirken. Energieschützend bedeutet in diesem Zusammenhang, dass weniger Energieträger verbraucht werden, wozu im Wesentlich drei Möglichkeiten bestünden: Es könnten weniger Energieträger für die gleiche Menge an Energiedienstleistungen in Anspruch genommen werden, was vor allem mit dem Einsatz energieeffizienter Geräte möglich wäre. Sogenannte nachhaltige Energieträger, wie Wind, könnten zur Deckung des restlichen Energiebedarfs verwendet werden. Und schließlich könnten generell weniger Energiedienstleitungen in Anspruch genommen werden, was letztlich einem Verzicht gleichkommen würde (Matthes, 2000). Diese drei Aspekte werden im Folgenden gemeint, wenn von Energieschutz (analog zu dem Begriff Umweltschutz) die Rede ist.

\subsection{Fragestellungen/Hypothesen}

Durch eine Übertragung auf das Handlungsfeld nachhaltigen Verhaltens soll überprüft werden, ob sich die Vermutung bestätigt, dass sich Rechtfertigungsargumente vor dem Hintergrund nachhaltigen Handelns abbilden lassen, und ob sie mit jenem Verhalten, aber auch der Attribution von Verantwortung in Verbindung stehen. Daraus ergeben sich die folgenden Untersuchungsfragen:

1. Inwiefern werden Rechtfertigungsargumente hervorgebracht, um Maßnahmen zur Einsparung von Energie abzuwenden und inwieweit wird somit die Verantwortung für diese Maßnahmen abgegeben?

Es ist davon auszugehen, dass Maßnahmen, die mit einer Einsparung von Energie einhergehen und sich somit langfristig positiv auf die Umwelt auswirken, keine uneingeschränkte Zustimmung in der Bevölkerung finden. Neben anderen möglichen Ursachen dafür wird angenommen, dass es verschiedene Argumente gibt, die weniger nach inhaltlichen Gesichtspunkten beurteilt werden, als vielmehr nach dem Kriterium, dass sie als Gründe für eine Ablehnung der Maßnahmen angeführt werden können und somit einen ausbleibenden Einsatz rechtfertigen. Ferner wird angenommen, dass sich diese Argumente entsprechend abbilden. 
2. Gibt es Zusammenhänge zwischen Rechtfertigungsargumenten und Verantwortungsattributionen?

Da Verantwortung ein zentrales Motiv auch innerhalb der Rechtfertigungsargumente darstellt, weil jene gewissermaßen als Wege aus der Verantwortung anzusehen sind, liegt es nahe, dass es Zusammenhänge zwischen diesem Konstrukt und verschiedenen Verantwortungsattributionen gibt. Dabei sollte sich vor allem ein negativer Zusammenhang zwischen der internalen Zuschreibung von Verantwortung und den Rechtfertigungsargumenten zeigen. Bei externalen Attributionen wird eine vergleichsweise geringere Korrelation erwartet.

3. Zeigen sich in der Äußerung von Rechtfertigungsargumenten Tendenzen für geschlechts-, bildungs- oder altersspezifische Unterschiede?

Auch wenn hierzu keine konkreten empirischen Befunde vorliegen, soll überprüft werden, ob etwaige Unterschiede existieren. Vor allem für zielgruppenspezifische Interventionen, beispielsweise im schulischen Kontext, könnte die Beantwortung dieser Frage entsprechende Anhaltspunkte liefern.

4. Hemmen Rechtfertigungsargumente energieschützende Engagement- und Handlungsbereitschaften?

Hierzu gibt es ebenfalls noch keine konkret auf dieses Handlungsfeld bezogene Untersuchung, die explizit die Auswirkung von Rechtfertigungsargumenten auf energierelevante Bereitschaften thematisiert. Vor dem Hintergrund dessen, was jedoch durch die bereits vorliegenden Studien bekannt ist (Schwartz \& Howard, 1981), liegt die Vermutung nahe, dass Rechtfertigung eine hemmende Wirkung auf den Energieschutz hat. Hingegen sollten die Bereitschaften, die mit dem Energieschutz interferieren, von Rechtfertigungsargumenten eher begünstigt werden.

\subsection{Untersuchungsvariablen}

Unter Berücksichtigung bisheriger Forschungsergebnisse wurde eine Skala zur Erfassung von Rechtfertigungsargumenten, im Sinne von Wegen aus der Verantwortung, entwickelt. Dabei fand eine Übertragung auf den Handlungsbereich Energie statt. Die Skala besteht aus sechs Items. Diese Items werden alle mit dem gleichen Satzanfang eingeleitet („Es liegt nicht in unserer Verantwortung, weitere Maßnahmen zu ergreifen, um Energie zu sparen, weil..."). Es folgen insgesamt sechs Argumente darauf, die eine Begründung liefern (Beispielitem: „... der Einzelne sowieso nichts ändern kann.“). Auch die übrigen fünf Statements umfassen 
ähnliche, letztlich vorgeschobene Gründe (Schwartz \& Howard, 1981), nicht für den Energieschutz aktiv zu werden. Dabei geht es nicht primär um die inhaltlichen Aspekte, sondern schlichtweg um die Rechtfertigung durch Argumente, die für den Einzelnen einigermaßen plausibel erscheinen und mittels derer er glaubt, sich aus der Verantwortung ziehen zu können. So geht es bei den Argumenten vor allem um die Rechtfertigung vor sich selbst, auch wenn der Grund dabei nur vorgeschoben ist. Die Probanden hatten die Möglichkeit, diesen Argumenten entsprechend zuzustimmen oder sie abzulehnen. Dies wiederum geschah über Ankreuzen auf einer sechsstufigen Likert-Skala. Die Antwortmöglichkeiten reichen von „trifft völlig zu“ bis „trifft überhaupt nicht zu“.

Weitere Verantwortungskognitionen wurden ebenfalls erfasst. Dies geschah in Form verschiedener Verantwortungszuschreibungen: zum einen die Attribution der Verantwortung auf die nächsten Generationen, daneben die Attribution auf Entwicklungs- und Schwellenländer, und schließlich auf sich selbst bzw. auf Deutschland. Die drei Dimensionen umfassen je drei Items, die sich jeweils auf Entscheidungsträger in Industrie und Wirtschaft, auf politische Entscheidungsträger sowie auf das Individuum beziehen. Eingeleitet wurden die insgesamt neun Items mit dem folgenden Satz: „Die Verantwortung für die Verringerung eines zu hohen Energieverbrauchs und den damit verbundenen Folgen und Risiken für die Umwelt und das Klima liegt bei...“. Item- und Skalenanlysen zeigen, dass sich diese zeitlich-räumlichen Aspekte hinsichtlich der Verantwortungszuschreibung klar herauskristallisieren. Auch hierzu hatten die Probanden das oben genannte Antwortformat vorliegen.

Darüber hinaus wurden verschiedene Handlungs- und Engagementbereitschaften abgefragt, ebenfalls über die sechsstufige Likert-Skala, um die Auswirkung von Rechtfertigungsargumenten auf energierelevante Engagement- und Handlungsbereitschaften zu untersuchen. Gegenstand der Untersuchung waren dabei Engagementbereitschaften, die darauf abzielen, Energie einzusparen (z. B. Maßnahmen zur Entwicklung neuer, energiesparender Technologien), aber auch jene, die mit Energieschutz potentiell in Konflikt stehen (z. B. die Senkung der KFZ-Steuer). Allerdings umfasst die zuletzt genannte Engagementbereitschaft wiederum andere persönliche und soziale Ziele, beispielsweise die Förderung eines hohen Lebensstandards, Arbeitsplatzsicherheit oder persönliche Entscheidungsfreiheit. Unter die Handlungsbereitschaften fallen zum einen die Bereitschaft zum Kauf umweltfreundlicher Produkte (z.B. Geräte, die teurer in der Anschaffung sind, aber weniger Energie verbrauchen), zum anderen die Bereitschaft zur Vermeidung energieintensiver Tätigkeiten im Alltag (z. B. der Verzicht des Einsatzes zeitsparender Haushaltsgeräte mit hohem Energieverbrauch, wie z. B. Wäschetrockner). Am Ende der Befragung wurden unter anderem noch soziodemographische Variablen wie Alter, Geschlecht, Beruf und Bildungsabschluss abgefragt. 


\subsection{Methode und Stichprobe}

Für die Untersuchung konnte insgesamt auf die Daten von 312 Personen aus der Allgemeinbevölkerung (156 Männer; 153 Frauen; drei ohne Angabe) zurückgegriffen werden, welche bei der Fragebogenerhebung teilnahmen. Die Stichprobe wurde über eine Zufallsauswahl rekrutiert, so erfolgte die Verbreitung des Fragebogens über verschiedene Onlineplattformen, E-Mailverteiler verschiedener Fachschaften und einen Presseaufruf der Universität Eichstätt-Ingolstadt. Insgesamt dauerte der Erhebungszeitraum zwölf Wochen an. Die befragten Personen waren zwischen 16 und 86 Jahre alt, mit einem Durchschnittsalter von 40,2 Jahren. Aufgrund der höheren Teilnahmebereitschaft von Menschen mit höherer Schulbildung sind Personen mit höherem Bildungsniveau in der vorliegenden Studie etwas überrepräsentiert. Gleichzeitig kann aber als sichergestellt angesehen werden, dass innerhalb der Studie ein breites Einstellungs- und Meinungsbild vertreten ist, da neben Vertretern der Allgemeinbevölkerung überdies auch noch Datenmaterial zweier Kriteriumsgruppen (Mitglieder von Umweltschutzorganisationen versus Motorsportclubs) in die Erhebung einging.

Die Güte des Messinstruments kann als gegeben angesehen werden: Die verwendeten Skalen bestehen aus mehreren Items, die das jeweilige Konstrukt abbilden, wobei umfangreiche Item- und Skalenanalysen die Reliabilität bestätigten. Ferner konnte mittels Extremgruppenvalidierung die Validität nachgewiesen werden. Sozial erwünschtes Antwortverhalten wurde durch verschiedene bewährte Strategien zu vermeiden versucht. Zudem fand die Skala zur Erfassung sozialer Erwünschtheit von Musch, Brockhaus \& Bröder (2002) Verwendung, so dass folglich sozial erwünschte Antworttendenzen erfolgreich kontrolliert werden konnten.

\section{$3 \quad$ Befunde}

Die neu entwickelte Rechtfertigungs-Skala umfasst, wie bereits erwähnt, verschiedene Argumente, die ein Nicht-Aktiv-Werden begründen. Bezugnehmend auf die erste Fragestellung konnte gezeigt werden, dass Rechfertigungsargumente durchaus vorgebracht werden, allerdings bewegen sich die Mittelwerte der einzelnen Items im Bereich zwischen $A M=1.79$ und $A M=2.94$, was darauf hindeutet, dass sie in der vorliegenden Stichprobe insgesamt eher gering ausgeprägt sind, folglich die Probanden tendenziell die Items der Skala und damit die Rechtfertigungsargumente eher ablehnen. Dennoch variieren die Ausprägungen innerhalb der Stichprobe ausreichend stark, was ebenfalls aus deskriptiven Kennwerten der Items, genauer 
gesagt den Standardabweichungen, die deutlich über 1 liegen, ersichtlich wird (vgl. Tabelle 1). Der Skalenmittelwert liegt bei $\mathrm{AM}=2.41, \mathrm{SD}=1.15$. Bemerkenswert ist vor allem, dass im Rahmen einer Hauptachsen-Faktorenanalyse, unter dem Kriterium Eigenwerte größer eins, alle Items auf einem Faktor laden, wobei die einzelnen Ladungen von .52 bis .78 reichen. Diese einfaktorielle Lösung wird auch durch den Screeplot nahegelegt. Cronbachs Alpha, das Maß für interne Konsistenz, ist mit einem Wert von .83 ebenfalls als zufriedenstellend anzusehen und spricht dafür, dass die Skala hinreichend reliabel ist.

Tab. 1 Deskriptive Kennwerte der Rechtfertigungsargumente

\begin{tabular}{|c|c|c|c|}
\hline Itemwortlaut & $\mathbf{N}$ & AM & SD \\
\hline \multicolumn{4}{|l|}{$\begin{array}{l}\text { Es liegt nicht in unserer Verantwortung, weitere Maßnahmen zu } \\
\text { ergreifen, um Energie zu sparen, weil... }\end{array}$} \\
\hline $\begin{array}{l}\text {... Deutschland bereits eine Vorreiterrolle im Hinblick auf Klima- } \\
\text { schutz und erneuerbare Energien einnimmt. }\end{array}$ & 309 & 2.65 & 1.55 \\
\hline ... der Einzelne sowieso nichts ändern kann. & 282 & 2.00 & 1.35 \\
\hline $\begin{array}{l}\text {... die Zusammenhänge des Klimawandels sehr komplex sind und } \\
\text { daher der Anteil, der auf Menschen zurückgeht unklar ist. }\end{array}$ & 310 & 2.37 & 1.46 \\
\hline $\begin{array}{l}\text {.. die globale Erwärmung trotzdem erstmal fortschreiten würde, } \\
\text { da das Klima nur sehr langsam auf Veränderungen reagiert. }\end{array}$ & 309 & 2.69 & 1.74 \\
\hline $\begin{array}{l}\text {... die anderen Industrienationen auch erst mal aktiv werden } \\
\text { müssen. }\end{array}$ & 309 & 2.94 & 1.90 \\
\hline ... es wichtigere Angelegenheiten gibt. & 307 & 1.79 & 1.15 \\
\hline
\end{tabular}

$\mathrm{N}=$ Anzahl der Personen, die zu diesem Item eine Angabe machen; AM = Mittelwert; SD = Standardabweichung, 1 = stimmt überhaupt nicht; 6 = stimmt genau

Da Rechtfertigungsargumente gewissermaßen "Wege aus der Verantwortung" darstellen, liegt es nahe, dass diese in negativer Weise mit der internalen Verantwortungsattribution in Zusammenhang stehen. Kein oder nur ein geringer Zusammenhang wird hingegen zwischen den Rechtfertigungsargumenten und der externalen Verantwortungsattribution erwartet. Diese zweite Fragestellung konnte mittels korrelativer Analysen überprüft werden. Befunde sprechen für die Annahme der Hypothese: Internale Verantwortungszuschreibung, im Sinne von Zuschreibung auf Deutschland, zeigt einen hochsignifikanten, negativen Zusammenhang $\left(r=-.39^{* *}\right)$. Die eher external ausgerichteten Attributionen hingegen, die sich auf zukünftige Generationen $\left(\mathrm{r}=-.12^{\star}\right)$ oder auf Entwicklungs- und Schwellenländer $\left(r=-.14^{*}\right)$ bezogen, weisen zwar auf dem $5 \%$ Niveau eine signifikante, aber nur sehr gering ausgeprägte Korrelation auf (vgl. Tabelle 2). 
Tab. 2 Korrelationen zwischen Rechtfertigungsargumenten und der Zuschreibung von Verantwortung

\begin{tabular}{ll}
\hline Verantwortungszuschreibung auf zukünftige Generationen & $-.12^{\star}$ \\
\hline Verantwortungszuschreibung auf Entwicklungs- und Schwellenländer & $-.14^{\star}$ \\
\hline Verantwortungszuschreibung auf Deutschland & $-.39^{\star *}$ \\
\hline
\end{tabular}

${ }^{\star} .01<\mathrm{p}<.05,{ }^{\star *} \mathrm{p}<.01$

Die dritte Untersuchungsfrage befasst sich mit einem möglichen Zusammenhang zwischen Rechtfertigungsargumenten und soziodemographischen Variablen. Hierzu wurden ebenfalls Korrelationen berechnet. Die Ergebnisse zeigen, dass vor allem zwischen Alter und Rechtfertigungsargumenten, aber auch zwischen Bildungsabschluss und Rechtfertigungsargumenten ein Zusammenhang festgestellt werden kann, wenn auch ein geringer, aber mit $r=.19^{\star *}$ und $r=-.19^{\star *}$ dennoch statistisch hochsignifikanter Zusammenhang. Die Richtung dieses Zusammenhangs ist dahingehend, dass Rechtfertigungsargumente bei älteren Probanden tendenziell höher ausgeprägt sind als bei jüngeren Probanden. Und bei Personen mit höheren Bildungsabschlüssen sind niedriger ausgeprägte Rechtfertigungsargumente zu erkennen als bei solchen mit niedrigeren Abschlüssen.

Ein weitaus kleinerer, aber immer noch signifikanter Zusammenhang konnte zwischen Rechtfertigungsargumenten und Geschlecht $\left(\mathrm{r}=-13 .^{*}\right)$ nachgewiesen werden, dahingehend, dass diese Argumente von Männern etwas stärker vertreten werden als von Frauen (vgl. Tabelle 3). Diese Befunde müssen jedoch sehr vorsichtig interpretiert werden, zumal keine Übertragung auf die Allgemeinbevölkerung möglich ist, sondern es handelt sich nur um gewisse Tendenzen, die sich innerhalb der Stichprobe gezeigt haben.

Tab. 3 Korrelationen zwischen Rechtfertigungsargumenten und ausgewählten soziodemographischen Variablen

\begin{tabular}{lc}
\hline & Rechtfertigungsargumente \\
\hline Alter & $.19^{* *}$ \\
\hline Geschlecht & $-.13^{* *}$ \\
\hline Bildungsabschluss & $-.19^{* *}$ \\
\hline
\end{tabular}

${ }^{\star} .01<\mathrm{p}<.05,{ }^{* *} \mathrm{p}<.01$

Um die vierte und letzte Untersuchungsfrage zu beantworten, wurden zunächst Korrelationsanalysen berechnet, die die Zusammenhänge zwischen den nachhaltigen 
Bereitschaften und Rechtfertigungsargumenten ermitteln. Tatsächlich deuten die Ergebnisse darauf hin, dass Rechtfertigungsargumente sowohl im privaten als auch im politischen Bereich umweltschützende Bereitschaften hemmen (vgl. Tabelle 4).

Tab. 4 Korrelationen von Rechtfertigungsargumenten und energierelevantem Engagement und Handlungsbereitschaften

\begin{tabular}{lc} 
& Rechtfertigungsargumente \\
\hline Bereitschaft zum Kauf neuer, energiesparender Geräte & $-.42^{* *}$ \\
\hline $\begin{array}{l}\text { Bereitschaft zur Vermeidung energieintensiver Tätigkeiten } \\
\text { im Alltag }\end{array}$ & $-.29^{* *}$ \\
\hline Engagementbereitschaft für den Energieschutz & $-.29^{* *}$ \\
\hline $\begin{array}{l}\text { Engagementbereitschaft für Maßnahmen, die mit dem } \\
\text { Energieschutz interferieren }\end{array}$ & $.54^{* *}$ \\
${ }^{*} .01<\mathrm{p}<.05,{ }^{* *} \mathrm{p}<.01$ &
\end{tabular}

Rechtfertigungsargumente haben einen negativen Einfluss auf die Bereitschaft, neue, energiesparende Geräte zu kaufen, aber auch darauf, energieintensive Tätigkeiten im Alltag zu vermeiden. Gleichzeitig hängen Rechtfertigungsargumente in negativem Maße mit der Bereitschaft zum Engagement für politische Maßnahmen zur Einsparung von Energie und somit zum Schutz des Klimas und der Umwelt zusammen. Ein hochsignifikanter positiver Zusammenhang hingegen zeigt sich zwischen Rechtfertigungsargumenten und der Engagementbereitschaft für mit dem Energieschutz interferierender Interessen, jene Verhaltensweisen, die zwar mit dem Energieschutz in Konflikt stehen, aber wiederum andere Ziele und Interessen vertreten, die durchaus auch bestimmte Werte in der Gesellschaft widerspiegeln.

Um die hemmende Auswirkung von Rechtfertigungsargumenten noch genauer $\mathrm{zu}$ untersuchen, und damit die Untersuchungsfrage endgültig beantworten zu können, wurde eine schrittweise multiple Regression von der Bereitschaft zum Kauf neuer, energiesparender Geräte auf die Rechtfertigungsargumente und die internale Verantwortungsattribution berechnet. Diese Bereitschaft wurde herausgegriffen, weil sich hier die höchste negative Korrelation mit den Rechtfertigungsargumenten zeigt. Außerdem wurde die internale Verantwortungszuschreibung miteinbezogen, von der ein positiver Einfluss auf die angeführte Bereitschaft zu erwarten ist. Das Ergebnis zeigt, dass sich beide Prädiktoren zur Vorhersage qualifizieren können (vgl. Tabelle 5). Insgesamt können $23 \%$ der Varianz aufgeklärt werden. Durch das Ergebnis wird außerdem noch einmal klar belegt, dass Rechtfertigungsargumente einen negativen Einfluss auf nachhaltige Bereitschaften haben, im konkreten Fall 
auf die Bereitschaft zum Kauf neuer, energiesparender Geräte, zumal der Regressionskoeffizient ein negatives Vorzeichen besitzt. Somit kann als belegt angesehen werden, dass die Zurückweisung von Verantwortung ein Hindernis für nachhaltige Handlungsbereitschaften darstellt.

Tab.5 Schrittweise multiple Regression der Bereitschaft zum Kauf neuer, energiesparender Geräte auf Rechtfertigungsargumente und internale Verantwortungsattribution

\begin{tabular}{llccccc}
\hline Kriterium & Prädiktoren & $\mathrm{R}^{2}$ & $\mathrm{~B}$ & $\mathrm{SE} \mathrm{B}$ & beta & $\mathrm{r}$ \\
\hline $\begin{array}{l}\text { Bereitschaft zum } \\
\begin{array}{l}\text { Kauf neuer energie- } \\
\text { sparender }\end{array}\end{array}$ & $\begin{array}{l}\text { Rechtfertigungsargumente } \\
\text { Geräte }\end{array}$ & .18 & $-.28^{\star *}$ & .05 & -.32 & -.42 \\
\hline $\begin{array}{l}\text { Verantwortungszuschreibung } \\
\text { auf Deutschland }\end{array}$ & .23 & $.30^{\star *}$ & .06 & .26 & .39 \\
\hline Konstante) & & & & & & \\
\hline
\end{tabular}

$\mathrm{F}_{\text {gesamt }}(2 / 307)=47.77^{\star *}$

${ }^{\star} .01<\mathrm{p}<.05 ;{ }^{* *} \mathrm{p}<.01$

\section{$4 \quad$ Diskussion und Ausblick}

Es konnte gezeigt werden, dass sich Rechtfertigungsargumente im Kontext nachhaltiger Verhaltensweisen, bezogen auf den Umgang mit Energie, abbilden. Die Skala besitzt zufriedenstellende Gütekriterien. Die Ausprägung in der vorliegenden Stichprobe ist nicht sehr hoch, aber die Probanden unterscheiden sich hinreichend voneinander. Möglicherweise könnte für künftige Forschung eine etwas weichere Formulierung mancher Items dazu führen, dass eine größere Zustimmung erreicht wird. Gleichzeitig muss darauf geachtet werden, dass die Items immer noch das Konstrukt entsprechend erfassen. Generell sollte dieser Aspekt bei der Replikation dieser Skala berücksichtigt werden.

Dass sich ein Verantwortungsaspekt hinter den Rechtfertigungsargumenten verbirgt, konnte ebenfalls durch die Befunde belegt werden. So korrelieren vor allem internale Verantwortungszuschreibung und Rechtfertigungsargumente hochsignifikant negativ $(r=-.39)$ miteinander, was bedeutet, dass hoch ausgeprägte Rechtfertigungsargumente mit niedriger internaler Verantwortungsattribution einhergehen und umgekehrt. Gleichzeitig lässt sich aber bei der multiplen Regression auch erkennen, dass sich Rechtfertigungsargumente im Bezug auf die Vorhersage nachhaltigen Verhaltens dennoch ausreichend von der Verantwortungsattributi- 
on unterscheiden. Beide können sich trotz der Korrelation untereinander in der schrittweisen multiplen Regression als signifikante Prädiktoren für den Kauf neuer energiesparender Geräte qualifizieren und klären unterschiedliche Varianzanteile auf. Ein möglicher Grund hierfür könnte sein, dass es sich bei der Verantwortungsattribution um eine Form moralischer Verantwortung handelt (Kals et al., 1998), während die Rechtfertigungsargumente als eher oberflächlich anzusehen sind (Schwartz \& Howard, 1981). Rechtfertigungsargumente beinhalten etwas Vorgeschobenes, nicht tiefer Reflektiertes, während Attribution von Verantwortung stärker moralisch ausgerichtet ist. Für künftige Forschung wäre es möglicherweise interessant, die Items zur internalen Verantwortungsattribution noch stärker auf die Ebene des Individuums zu beziehen.

Es wurden alters- und bildungsspezifische Zusammenhänge bei Rechtfertigungsargumenten entdeckt, die jedoch aufgrund der Stichprobengröße sehr vorsichtig zu beurteilen sind. Für eine vertiefende Analyse wäre ein weitaus größerer Stichprobenumfang zwingend erforderlich. Es war jedoch auch nicht das Ziel der vorliegenden Untersuchung, Aussagen auf die Allgemeinbevölkerung zu übertragen. Dennoch sind die zu erkennenden Tendenzen insofern spannend, als dass sie vor allem für zielgruppenspezifische Interventionen beispielsweise im schulischen Kontext entsprechende Anhaltspunkte liefern könnten. Demnach wäre es denkbar, dass je nach Schulart oder Klassenstufe Rechtfertigungsargumente unterschiedlich ausgeprägt sind, was jedoch noch weiter erforscht werden müsste.

Vor dem Hintergrund bisheriger Forschungsergebnisse ist anzunehmen, dass Rechtfertigungsargumente einen negativen Einfluss auf nachhaltiges Verhalten haben (van Liere \& Dunlap, 1978). Die Ergebnisse der vorliegenden Studie bestätigen dies, so konnte gezeigt werden, dass Verhalten durch eine Reihe von Rechtfertigungsargumenten gehemmt werden kann. Die Befunde geben letztlich eine Antwort darauf, weshalb Menschen nicht nachhaltig handeln, selbst wenn Gründe für ein Handeln eingesehen wurden. Es wird aber auch deutlich, dass es nicht alleine ausreicht, an die moralische Verantwortung der Zielpersonen zu plädieren, sondern dass auch die Zurückweisung der Verantwortung über die Rechtfertigungsargumente verhindert werden muss. Dies sollte vor allem im Hinblick auf mögliche Interventionen, mittels derer nachhaltiges Verhalten gefördert werden, bedacht werden. Hierzu mag die Formulierung von Gegenargumenten hilfreich sein oder die Außerkraftsetzung der Wirkmechanismen hinter diesen Rechtfertigungsargumenten, wie beispielsweise die mangelnden Selbstwirksamkeitserwartungen, indem die Einflussmöglichkeiten konkreter aufgezeigt werden. Auch ein Bewusstmachen des sehr komplexen Ursache-Wirkungsgefüges, das sich hinter der Klimaproblematik verbirgt, könnte sich, bezogen auf die Zurückweisung von Verantwortung, als vorteilhaft erweisen. 


\section{Literatur}

Bennett, S., Banyard, V. \& Garnhart, L. (2014). To act or not to act, that is the question? Barriers and facilitators of bystander intervention. Journal of Interpersonal Violence, 29: $476-496$.

Clayton, S. (2000). Models of justice in the environmental debate. Journal of Social Issues, 5: 459-474.

Darley, J. M. \& Batson, C.D. (1973). "From Jerusalem to Jericho": A study of situational and dispositional variables in helping behavior. Journal of Personality and Social Psychology, 27: $100-108$.

Darley, J. M. \& Latané, B. (1968). Bystander intervention in emergencies: Diffusion of responsibility. Journal of Personality and Social Psychology, 8: 377-383.

van Erp, K. J., Rispens, S., Gevers, J. M. \& Demerouti, E. (2015). When bystanders become bothersome: The negative consequences of bystander conflict and the moderating role of resilience. European Journal of Work and Organizational Psychology, 24: 402-419.

Fritsche, I., Cohrs, J. C., Kessler, T. \& Bauer, J. (2012). Global warming is breeding social conflict: The subtle impact of climate change threat on authoritarian tendencies. Journal of Environmental Psychology, 32: 1-10.

Gifford, R. (2007). Environmental psychology and sustainable development: Expansion, maturation, and challenges. Journal of Social Issues, 63: 199-212.

Girardet, H. \& Mendonça, M. (2010). Neue Energien freisetzen: Für eine ökologische und gerechte Welt. Zürich: Rotpunktverlag.

Haines, A., Ebi, K. L., Smith, K. R. \& Woodward, A. (2014). Health risks of climate change: Act now or pay later. The Lancet, 384: 1073-1075.

Hardin, G. (1968). The tragedy of the commons. Science, 162: 1243-1248.

Hunecke, M., Blöbaum, A., Matthies, E. \& Höger, R. (2001). Responsibility and environment: Ecological norm orientation and external factors in the domain of travel mode choice behavior. Environment and Behaviour, 33: 830-852.

Kals, E. (1996). Verantwortliches Umweltverhalten: Umweltschützende Entscheidungen erklären und fördern. Weinheim: Psychologie Verlags Union.

Kals, E., Montada, L., Becker, R. \& Ittner H. (1998). Verantwortung für den Schutz von Allmenden. GAIA, 7: 296-303.

Kazdin, A. E. (2009). Psychological science's contributions to a sustainable environment: Extending our reach to a grand challenge of society. American Psychologist, 64(5): 339-356.

Klöckner, C. A. \& Blöbaum, A. (2010). A comprehensive action determination model: Toward a broader understanding of ecological behaviour using the example of travel mode choice. Journal of Environmental Psychology, 30: 574-586.

Latané, B. \& Rodin, J. (1969). A lady in distress: Inhibiting effects of friends and strangers on bystander intervention. Journal of Experimental Social Psychology, 5: 189-202.

van Liere, K. D. \& Dunlap, R. E. (1978). Moral norms and environmental behavior: An application of Schwartz's norm-activation model to yard burning. Journal of Applied Social Psychology, 8: 174-188.

Linneweber, V. (1995). Energienutzung in Privathaushalten: ein feldexperimenteller Vergleich von Interventionsstrategien. Zeitschrift für Experimentelle Psychologie, 42: 455-490.

Linneweber, V. (1998). „Nachhaltige Entwicklung“ als unscharfes Prädikat. Umweltpsychologie 2: 66-76. 
Matthes, F. C. (2000). Strukturelle Bedingungen nachhaltigen Energiekonsums. In: C. Günther, C. Fischer \& S. Lerm (Hrsg.), Neue Wege zu nachhaltigem Konsumverhalten. Eine Veranstaltung der Deutschen Bundesstiftung Umwelt zur EXPO 2000 (Band 22) (pp. 105-110). Berlin: Erich Schmidt.

Müller, M. M. (2012). Justice as a framework for the solution of environmental conflicts. In: E. Kals \& J. Maes (Hrsg .), Justice and conflicts. Theoretical and empirical contributions (pp. 239-250). Berlin: Springer.

Musch, J., Brockhaus, R. \& Bröder, A. (2002). Ein Inventar zur Erfassung von zwei Faktoren sozialer Erwünschtheit. Diagnostica, 48: 121-129.

Nancarrow, B. E. \& Syme, G. J. (2001). Challenges in implementing justice research in the allocation of natural resources. Social Justice Research, 14: 441-452.

Pawlik, K. (1991). The psychology of global environmental change: Some basic data and an agenda for cooperative international research. International Journal of Psychology, 26: 547-563.

Pittinsky, T. L. \& Diamante, N. (2015). Global bystander nonintervention. Peace and Conflict: Journal of Peace Psychology, 21: 226-247.

Plötner, M., Over, H. \& Tomasello, M. (2015). Young children show the bystander effect in helping situations. Psychological Science, 26: 499- 506.

Raffa, R. B., Eltoukhy, N. S. \& Raffa, K. F. (2012). Implications of climate change (global warming) for the healthcare system. Journal of Clinical Pharmacy and Therapeutics, 37: 502-504.

Schwartz, S. H. \& Clausen, G. T. (1970). Responsibility, norms, and helping in an emergency. Journal of Personality and Social Psychology, 16: 299-310.

Schwartz, S. H. \& Howard, J. A. (1981). A normative decision-making model of altruism. In: J. P. Rushton \& R. Sorrentino (Hrsg.), Altruism and helping behavior: Social, personality and development perspectives (pp. 189-211). Hillsdale: Erlbaum.

Schwartz, S. H. (1977). Normative influence on altruism. Advances in Experimental Social Psychology, 10: 221-279.

Spada, H. \& Opwis, K. (1985). Ökologisches Handeln im Konflikt: Die Allmende-Klemme. In: P. Day, U. Fuhrer \& U. Laucken (Hrsg.), Umwelt und Handeln (pp. 63-85). Tübingen: Attempto.

Stern, P. C. (2011). Contributions of psychology to limiting climate change. American Psychologist, 66: 303-314.

World Wide Fund For Nature (o. J.). Die Ursachen des Klimawandels. http://www.wwf.de/ themen-projekte/klima-energie/klimawandel/die-verursacher. Zugegriffen: 05.11.2015.

Wortmann, K. (2004). Energie als Thema der Umweltpsychologie: Einführung in das Schwerpunktthema. Umweltpsychologie, 8: 2-11. 
Open Access Dieses Kapitel wird unter der Creative Commons Namensnennung - Nicht kommerziell 2.5 International Lizenz (http://creativecommons.org/licenses/by-nc/2.5/deed. de) veröffentlicht, welche für nicht kommerzielle Zwecke die Nutzung, Verbreitung und Wiedergabe in jeglichem Medium und Format erlaubt, sofern Sie den/die ursprünglichen Autor(en), den Titel des Werks und die Quelle ordnungsgemäß nennen, einen Link zur Creative Commons Lizenz beifügen und im Falle einer Abwandlung durch einen entsprechenden Hinweis deutlich erkennbar machen, dass Änderungen vorgenommen wurden.

Die in diesem Kapitel enthaltenen Bilder und sonstiges Drittmaterial unterliegen ebenfalls der genannten Creative Commons Lizenz, sofern sich aus der Abbildungslegende nichts anderes ergibt. Sofern das betreffende Material nicht unter der genannten Creative Commons Lizenz steht und die betreffende Handlung nicht nach gesetzlichen Vorschriften erlaubt ist, ist auch für die oben aufgeführten nicht-kommerziellen Weiterverwendungen des Materials die Einwilligung des jeweiligen Rechteinhabers einzuholen. 